\title{
Cost Allocation of Capacity Investment Games
}

\author{
Xin Chen, ${ }^{1}$ Zhisong Chen ${ }^{2}$ \\ ${ }^{1}$ Department of Industrial and Enterprise Systems Engineering, University of Illinois at Urbana-Champaign, \\ Urbana, Illinois \\ ${ }^{2}$ Business School, Nanjing Normal University, Nanjing, Jiangsu, China
}

Received 15 December 2011; revised 22 June 2013; accepted 23 June 2013

DOI 10.1002/nav.21549

Published online 5 August 2013 in Wiley Online Library (wileyonlinelibrary.com).

\begin{abstract}
Consider a manufacturer serving a set of retail stores each of which faces deterministic demands in a finite planning horizon. At the beginning of the planning horizon, the production capacity of the manufacturer is built, followed by production, outsourcing to third party manufacturers if necessary and distribution to the retail stores. Because the retail stores are usually managed by different managers who act as independent profit centers, it is desirable that the total cost is divided among the retail stores so that their incentives can be appropriately captured and thus efficient operations can be achieved. Under various conditions, we prove that there is a fair allocation of costs among the retail stores in the sense that no subset of retail stores subsidizes others, or equivalently, the resulting capacity investment game has a nonempty core, that is, the capacity investment game is a balanced game. In addition, our proof provides a mechanism to compute a fair cost allocation. (c) 2013 Wiley Periodicals, Inc. Naval Research Logistics 60: 512-523, 2013
\end{abstract}

Keywords: cooperative game; quantity discount; cost allocation; capacity

\section{INTRODUCTION}

Cost allocation plays a critical role in a variety of settings in which common facilities or resources are built, used, and/or maintained by decentralized decision makers (see Young [21]). For example, towns using a common water supply system need to split the construction and maintenance costs (see Young et al. [22]); receivers of multicast transmissions share the bandwidth and thus have to distribute their charges (see Feigenbaum et al. [12]); different market agents in the power industry allocate costs for using transmission and distribution networks (see Zolezzi and Rudnick [25]).

It has also been long recognized that common costs within a firm should be distributed in a way that appropriately reflects the burden each division exerted on others. Indeed, poor allocation may lead to distorted incentives and result in inefficient operations (see Shubik [15]). Though its importance is well-understood and various cost allocation mechanisms have been proposed in the managerial accounting literature (see Young [21]), it is nevertheless nontrivial to allocate costs so as to give the division managers the right incentives to achieve efficient operations. One of the challenges is that

Correspondence to: X. Chen (xinchen@illinois.edu) common cost is usually not given as a prior. Instead, it may heavily depend on firms' operational decisions.

In this article, we analyze cost allocation in a manufacturing setting in which a firm produces a single product to serve its retail stores at different locations in a finite planning horizon. At the beginning of the planning horizon, the production facility is built and its capacity is determined. The firm then makes production decisions so as to meet the demands of the retail stores. The demand of each retail store is assumed to be deterministic and must be met without backlogging. If the capacity is not sufficient to meet all demands, the firm may outsource from subcontractors. The objective of the firm is to meet the demands of the retail stores while minimizing its total costs, including capacity investment cost, production costs, outsourcing costs, inventory holding costs at both the production facility, and the retail stores and transportation costs to the retail stores.

Because the retail stores are usually managed by different managers who act as independent profit centers, the firm needs to allocate the minimal cost of running the common production facility to them without distorting their cost implications on the system. Specifically, it is appropriate to allocate the cost fairly among the retail stores in the sense that no subset of retail stores will subsidize others. The notion of fairness can be captured by the concept of core in cooperative game 
theory. Roughly speaking, a cost allocation in the core implies that no subset of players would benefit by leaving the whole group under this cost allocation, which equivalently implies that no subset of players subsidizes others. Thus, we model the firm's cost allocation problem as a cooperative game, referred to as the capacity investment game, and investigate whether there exist cost allocations in the core of the game.

The above setting is also appropriate for modeling third party logistics providers providing transportation services for several parties in which delivery requests in excess of capacities can be outsourced to other contracted carriers by charging a higher rate (see Atamtürk and Hochbaum [3]). Accurate cost allocation is critical for third party logistics providers to identify the true costs of services.

The main contribution of this article is to show that the core of the capacity investment game is nonempty under various conditions and develop a mechanism to compute a cost allocation in the core. Specifically, when all related costs are linear, we use linear programming duality to construct an allocation in the core of the capacity investment game. The result will then be used to compute core allocations for games with more general capacity investment cost functions. We comment that it is common to assume linear inventory holding costs and linear transportation costs in the operations literature though other cost structures are also possible (see Simchi-Levi et al. [16]).

We then focus on more general capacity investment cost functions which exhibit the quantity discount property, that is, the larger the capacity level, the lower the average unit capacity investment cost. Under these capacity investment costs, two different cases are analyzed. In the first case, we assume that no inventory is carried over from one period to the next at either the production facility or the retail stores. Under this assumption, we prove that the core is nonempty if the production costs exhibit the quantity discount property as well while the outsourcing costs are linear (the transportation costs to the retail stores are constant in this case and thus can be ignored in the analysis). On the other hand, the core may be empty if the outsourcing costs fail to be linear. The assumption of no inventory carryover is appropriate for settings in which the focus is on "balancing the tradeoff between capacity acquisition and subcontracting in telecommunication as well as in other service industries where holding inventory is not an option" (see Atamtürk and Hochbaum [3]). In this case, one can interpret the production facility as a central service facility and different retail stores as service requests for different players.

In the second case, inventory is allowed to be carried from one period to the next at both the production facility and the retail stores. We show that the core is nonempty if all costs other than the the capacity investment cost are linear. Our assumption of linear production costs is appropriate for settings in which economies of scale of production are negligible. It is also appropriate for settings in which machine setup costs or setup times are so significant that machine shutdown is prohibited. For instance, a glass manufacturing company we have been working with essentially never shuts down its machines because for each setup it takes a month for the company to calibrate the temperature settings of its furnaces for normal glass production. In this case, it is sufficient to focus on linear production costs only.

Our approach to the capacity investment game with general capacity cost builds on a general technique developed in Chen [5] for inventory centralization games. The idea is to construct a new game with linear capacity investment cost, which is known to have a nonempty core, such that the minimum cost achieved by any subset of the retail stores in the new game will not decrease, while ensuring the minimum cost achievable by all retail stores remains unchanged (we say the new game is dominated by the original game). In addition to the proof of the nonemptiness of the core, our approach also suggests a mechanism to find an allocation in the core for the capacity investment game with a general capacity cost. To apply our approach, we prove that the optimal capacity investment level is nondecreasing in terms of the demand vectors of the retail stores for both cases with or without inventory carryover.

Our research is related to economic lot sizing games analyzed by Tamir [17], van den Heuvel et al. [19], and Chen and Zhang [6]. In these papers, different retailers place joint orders and share common inventory. Under certain conditions, they prove that economic lot sizing games admit nonempty cores. However, in their settings, ordering quantities are uncapacitated and no capacity investment is modeled, whereas in our article capacity investment is critical and presumably more expensive than ordering costs. These papers belong to the growing literature on inventory centralization games; see Anily and Haviv [1], Chen [5], Chen and Zhang [7], Zhang [24], Xu and Yang [20], and Gopaladesikan et al. [9] for recent progress. Guardiola et al. [11] propose a class of production-inventory games derived from combinatorial optimization problems. We refer to Curiel [8] for more cooperative games arising from combinatorial optimization problems, and Nagarajan and Sošić [13] for a comprehensive review of applications of cooperative game to supply chain settings.

Our article is also closely related to Yu et al. [23] and Anily and Haviv [2], who analyze the benefit of capacity pooling and its associated cost allocation problem among independent firms. In their paper, each firm may operate its own facility modeled as an $M / M / 1$ queue or the firms can invest in a shared facility again modeled as an $M / M / 1$ queue. Similar to our article, the capacity of the shared facility, that is, the service rate of the server in the queue, is endogenously determined. However, in their paper, the benefit of cooperation results from risk pooling, whereas in our article, the benefit 
comes from taking advantage of economies of scale. Like our paper, Anily and Haviv [2] construct new games to facilitate their analysis. However, it serves a different purpose. Specifically, although we construct new games to prove the existence of core allocations and provide a mechanism to find one core allocation, Anily and Haviv [2] construct new games to identify all core allocations with nonnegative entries.

The underlying optimization problem in our capacity investment game is similar to the capacity acquisition model analyzed by Atamtürk and Hochbaum [3]. In their model, capacity is installed at the beginning of the finite planning horizon, which is followed by production and subcontracting during the planning horizon. Unlike our article, they focus on a centralized system with a single-decision maker and develop polynomial time algorithms under a variety of conditions on the costs.

The organization of this article is as follows. In Section 2 , we first present our capacity investment game model. In Section 3 , we use convex programming duality theory to prove the nonemptiness of the core when the costs at the production facility are all linear. The case with possibly nonlinear costs at the production facility is analyzed in Section 4, which is followed by some concluding remarks in Section 5 .

Before proceeding to introduce our capacity investment game model, we briefly introduce the basic concepts of cooperative game theory that will be used in this article.

Let $N=\{1,2 \ldots, n\}$ be the set of players. A collection of players $S \subseteq N$ is called a coalition. The set $N$ is sometimes referred to as the grand coalition. A characteristic cost function $C(S)$ is defined for each coalition $S \subseteq N$, which could be the minimum total cost that coalition $S$ incurs if the members of $S$ decide to cooperate among themselves. A cooperative game is determined by the pair $(N, C)$. The game $(N, C)$ is called subadditive if for every pair of subsets $S, T \subseteq N$ with $S \cap T=\emptyset, C(S \cup T) \leq C(S)+C(T)$. It is called a concave game if for every pair of subsets $S, T \subseteq N$, $C(S)+C(T) \geq C(S \cup T)+C(S \cap T)$, or equivalently, $C(S)$ is submodular in $S$.

A vector $\mathbf{l}=\left(l_{1}, l_{2}, \ldots, l_{N}\right)$ is called an efficient allocation for the game $(N, C)$ if $\sum_{j \in N} l_{j}=C(N)$. The core of a cooperative game is a solution concept which requires that no subset of players has an incentive to secede.

DEFINITION 1: An allocation $\mathbf{I}$ is in the core of the game $(N, C)$, if $\sum_{j \in N} l_{j}=C(N)$ and for any subset $S \subseteq N$, $\sum_{j \in S} l_{j} \leq C(S)$.

A game $(N, C)$ is called balanced if it has a nonempty core. Observe that $C(N) \leq C(S)+C(N \backslash S)$ for any $S \subseteq N$ if $(N, C)$ is a balanced game. In addition, the above definition is equivalent to saying that for any coalition $S \subseteq N$, $\sum_{j \in S} l_{j} \geq C(N)-C(N \backslash S)$. This implies that the total cost paid by players in $S$ is sufficient to compensate the cost imposed by adding the coalition to the grand coalition, or equivalently no coalition is subsidized by other players outside the coalition.

The concept of core involves an exponential number of linear inequalities. Thus, in general, it is not trivial to show whether the core of a cooperative game is empty or not and to find an allocation in the core (if nonempty) efficiently.

\section{THE CAPACITY INVESTMENT GAME MODEL}

Consider a firm with a set of $n$ retail stores denoted by $N=$ $\{1,2, \ldots, n\}$. Each retail store faces deterministic demands over a finite horizon with a total of $T$ periods. Let $d_{t}^{j}$ be the demand of retail store $j$ at period $t$ and $\mathbf{d}^{j}=\left(d_{1}^{j}, \ldots, d_{T}^{j}\right)$ be its demand vector. To satisfy the demands of the retail stores, the firm needs to build a common production capacity at the beginning of the planning horizon, which incurs a capacity investment cost $v(z)$ for a capacity level $z$. After the production capacity is built, the firm decides how much to produce and how much demand to be outsourced, that is, satisfied by an outside producer, at each period. The production quantity at each period cannot exceed the production capacity and no backorder is allowed. At period $t$, the production cost and the outsourcing cost are given respectively by $c_{t}\left(x_{t}\right)$ and $o_{t}\left(y_{t}\right)$ if the production quantity is $x_{t}$ and the outsourcing level is $y_{t}$.

The products produced at the production facility and outsourced from the outside producer are stored at the production facility as inventory, which can be transhipped to the retail stores with a transportation cost $u_{t}^{j}\left(q_{t}^{j}\right)$ if the quantity of the product transported from the production facility to retail store $j$ is $q_{t}^{j}$. On the other hand, inventories at the production facility and the retail stores may also be carried over from period $t$ to period $t+1$ at a $\operatorname{cost} h_{t}^{j}\left(I_{t}^{j}\right)$, where $I_{t}^{j}$ is the inventory level at retail store $j$ (the product facility if $j=0$ ) at the end of period $t$ (observe that the case with no inventory carryover can be modeled by setting very high inventory costs). The objective of the firm is to find a capacity level and a production and distribution plan so as to minimize the total cost, including capacity investment cost, production cost, outsourcing cost, inventory holding cost, and transportation cost.

Given the demand profile $\mathbf{D}=\left(\mathbf{d}^{1}, \mathbf{d}^{2}, \ldots, \mathbf{d}^{n}\right)$, the minimal cost of the firm is given by $f(\mathbf{D})$ with

$$
f(\mathbf{D})=\min _{z \geq 0} v(z)+g(z, \mathbf{D}),
$$

where

$$
\begin{aligned}
g(z, \mathbf{D})= & \min \sum_{t=1}^{T}\left\{c_{t}\left(x_{t}\right)+o_{t}\left(y_{t}\right)+h_{t}^{0}\left(I_{t}^{0}\right)\right. \\
& \left.+\sum_{j \in N}\left[h_{t}^{j}\left(I_{t}^{j}\right)+u_{t}^{j}\left(q_{t}^{j}\right)\right]\right\}
\end{aligned}
$$




$$
\begin{array}{ll}
\text { s.t. } & I_{t}^{0}=I_{t-1}^{0}+x_{t}+y_{t}-\sum_{j \in N} q_{t}^{j}, \\
& t=1,2, \ldots, T, \\
& x_{t}-z \leq 0, \quad t=1,2, \ldots, T, \\
& I_{0}^{0}=0, \\
& I_{t}^{0} \geq 0, \quad t=1,2, \ldots, T, \\
& x_{t}, y_{t} \geq 0, \quad t=1,2, \ldots, T, \\
& \left(\mathbf{I}^{j}, \mathbf{q}^{j}\right) \in X^{j}, j \in N,
\end{array}
$$

and

$$
\begin{array}{r}
X^{j}=\left\{\left(\mathbf{I}^{j}, \mathbf{q}^{j}\right) \mid I_{t}^{j}=I_{t-1}^{j}+q_{t}^{j}-d_{t}^{j}, I_{t}^{j} \geq 0, q_{t}^{j} \geq 0 \forall t\right. \\
\left.=1,2, \ldots, T, I_{0}^{j}=0\right\} .
\end{array}
$$

In the above problem, the objective function involves the capacity investment $\operatorname{cost} v(z)$, the production $\operatorname{costs} c_{t}\left(x_{t}\right)$, the outsourcing costs $o_{t}\left(y_{t}\right)$, the inventory holding costs $h_{t}^{0}\left(I_{t}^{0}\right)$ at the production facility, and the transportation costs $u_{t}^{j}\left(q_{t}^{j}\right)$ to the retail stores, and the inventory holding costs $h_{t}^{j}\left(I_{t}^{j}\right)$ at the retail stores. The first constraint is the inventory balance equation at the production facility, the second constraint is the capacity constraint, and $X^{j}$ specifies the inventory balance equations at retail store $j$. We also assume that no backorder is allowed at either the production facility or any retail store, which corresponds to the inequalities $I_{t}^{j} \geq 0$.

It is appropriate to point out that the above optimization problem is similar to the capacity acquisition model analyzed by Atamtürk and Hochbaum [3]. The difference is that in their model there is only a single-retail store which coincides with the production facility. It is straightforward to see that their model corresponds to our case with no inventory carryover at the retail stores. In addition, their focus is on developing efficient algorithms for solving the capacity acquisition model, whereas we are interested in the cost allocation among the retail stores.

Because the retail stores are usually managed by different managers which act as independent profit centers, it is critical that the total cost is allocated fairly among the retail stores to appropriately capture the burden each retail store imposes on the common production facility. As we already pointed out in the introduction, fairness can be modeled using the concept of core in an appropriately constructed cooperative game. In our setting, the cooperative game, referred to as the capacity investment game, can be specified by $(N, C)$ where the grand coalition $N$ is the set of retail stores and the characteristic cost function $C(S)$ is given by the minimum cost for coalition $S$ when the coalition builds a production facility and makes production and distribution decisions together to satisfy the demand of the coalition members. Specifically, the minimum cost for coalition $S, C(S)$, can be written as

$$
C(S)=f(\mathbf{D}(S)),
$$

where $\mathbf{D}(S)=\left(\mathbf{d}^{1}(S), \mathbf{d}^{2}(S), \ldots, \mathbf{d}^{n}(S)\right)$ with

$$
\mathbf{d}^{j}(S)= \begin{cases}\mathbf{d}^{j}, & \text { if } j \in S \\ 0, & \text { otherwise. }\end{cases}
$$

It is not hard to construct examples to show that if $v(\cdot), c_{t}(\cdot), o_{t}(\cdot)$, and/or $h_{t}^{0}(\cdot)$ are convex, that is, there are diseconomies of scale at the production facility, $C(S)$ may not be subadditive and the core of the cooperative game $(N, C)$ may be empty. For instance, consider a simple model in which no inventory carryover is allowed at the retail stores. In addition, the transportation cost is assumed to be zero. If $c_{t}(\cdot)=0$ and $o_{t}(\cdot)$ and $h_{t}^{0}(\cdot)$ are linear with large coefficients, then in this case, it is optimal for coalition $S$ to set $z(S)=\max _{t=1,2, \ldots, T} d_{t}^{S}, x_{t}=d_{t}^{S}, y_{t}=0$ and $I_{t}^{0}=0$, where $d_{t}^{S}=\sum_{j \in S} d_{t}^{j}$. Therefore, $C(S)=v(z(S))$. Given two nonempty coalitions $S$ and $S^{\prime}$ with $S \cap S^{\prime}=\emptyset$, if the largest aggregate demands of the two coalitions $S$ and $S^{\prime}$ occur at the same period, we have that $z(S)+z\left(S^{\prime}\right)=z\left(S \cup S^{\prime}\right)$. If $v(\cdot)$ is strictly convex and $v(0)=0$, then we have $C\left(S \cup S^{\prime}\right)>C(S)+C\left(S^{\prime}\right)$ unless $z(S)=0$ or $z\left(S^{\prime}\right)=0$. Hence, $C(S)$ may fail to be subadditive. If in addition, $S^{\prime}=N \backslash S$, then $(N, C)$ has an empty core. Similar examples can also be constructed if $c_{t}(\cdot), o_{t}(\cdot)$ and/or $h_{t}^{0}(\cdot)$ are convex.

The following example illustrates that the capacity investment game is not concave in general even when all costs are linear. Specifically, we look at a two-period model with no outsourcing. We assume that both the production facility and the retail stores do not hold inventory, or equivalently the inventory holding costs are extremely large. In addition, the production cost and the transportation cost are zero, and the capacity installation cost is 1 per unit. Let the total demand at period one be $d_{1}$ and the total demand at period two be $d_{2}$. It is clear that the optimal capacity level and hence the total cost are given by $\max \left(d_{1}, d_{2}\right)$. Assume that $S=\{1,2\}$, $S^{\prime}=\{1,3\}$ and $d^{1}=(0,1), d^{2}=d^{3}=(1,0)$. Then

$$
C(S)+C\left(S^{\prime}\right)=2<3=C\left(S \cap S^{\prime}\right)+C\left(S \cup S^{\prime}\right) .
$$

Therefore, the game is not concave.

Interestingly, any core allocation, if exists, does not contain negative entries, that is, no retail store will be paid to cooperative with the rest. To see this, consider any core allocation $\left(l_{1}, l_{2}, \ldots, l_{n}\right)$ of a game $(N, C)$. As for any $i \in N$, $\sum_{j \in N} l_{j}=C(N)$ and $\sum_{j \in N \backslash\{i\}} l_{j} \leq C(N \backslash\{i\})$, we have that $l_{i} \geq C(N)-C(N \backslash\{i\}) \geq 0$.

\section{LINEAR COSTS}

In this section, we assume that all costs are linear. The assumption is appropriate for settings in which economies of scale of production are negligible. More importantly, this section will serve as the basis for the analysis in the next 
section. By abusing notations, we denote $v(z)=v z, c_{t}\left(x_{t}\right)=$ $c_{t} x_{t}, o_{t}\left(y_{t}\right)=o_{t} y_{t}, h_{t}^{0}\left(I_{t}^{j}\right)=h_{t}^{j} I_{t}^{j}$ and $u_{t}^{j}\left(q_{t}^{j}\right)=u_{t}^{j} q_{t}^{j}$. In this case, problem (1) is a linear programming problem.

We now apply linear programming duality theory to problem (1). Define the Lagrangian function

$$
\begin{aligned}
& L_{S}(z, \mathbf{x}, \mathbf{y}, \mathbf{I}, \mathbf{q}, \lambda, \mu, \pi, \phi, \psi) \\
= & v z+\sum_{t=1}^{T}\left\{c_{t} x_{t}+o_{t} y_{t}+h_{t}^{0} I_{t}^{0}+\sum_{j \in S}\left[h_{t}^{j} I_{t}^{j}+u_{t}^{j} q_{t}^{j}\right]\right\} \\
& +\sum_{t=1}^{T} \lambda_{t}\left[I_{t}^{0}-\left(I_{t-1}^{0}+x_{t}+y_{t}-\sum_{j \in S} q_{t}^{j}\right)\right] \\
& +\sum_{t=1}^{T} \mu_{t}\left(x_{t}-z\right)+\sum_{t=1}^{T} \pi_{t}\left(-I_{t}^{0}\right) \\
& +\sum_{t=1}^{T}\left[\phi_{t}\left(-x_{t}\right)+\psi_{t}\left(-y_{t}\right)\right] \\
= & \left(v-\sum_{t=1}^{T} \mu_{t}\right) z+\sum_{t=1}^{T}\left[c_{t}-\lambda_{t}+\mu_{t}-\phi_{t}\right] x_{t} \\
& +\sum_{t=1}^{T}\left[o_{t}-\lambda_{t}-\psi_{t}\right] y_{t} \\
& +\sum_{t=1}^{T}\left[h_{t}^{0}+\left(\lambda_{t}-\lambda_{t+1}-\pi_{t}\right)\right] I_{t}^{0} \\
& +\sum_{t=1}^{T} \sum_{j \in S}\left[h_{t}^{j} I_{t}^{j}+u_{t}^{j} q_{t}^{j}+\lambda_{t} q_{t}^{j}\right] \\
& +{ }_{t}{ }_{t} \\
&
\end{aligned}
$$

where $\lambda_{T+1}=0$, and $\left(\mathbf{I}^{j}, \mathbf{q}^{j}\right) \in X^{j}, j \in S$.

Consider the dual function $\gamma_{S}(\lambda, \mu, \pi, \phi, \psi)$ defined by

$$
\begin{aligned}
\gamma_{S}(\lambda, \mu, \pi, \phi, \psi)=\min & L_{S}(z, \mathbf{x}, \mathbf{y}, \mathbf{I}, \mathbf{q}, \lambda, \mu, \pi, \phi, \psi) \\
\text { s.t. } & \left(\mathbf{I}^{j}, \mathbf{q}^{j}\right) \in X^{j}, j \in S .
\end{aligned}
$$

The linear programming strong duality theorem implies that $C(S)$ is equal to the optimal objective value of the dual problem:

$$
\begin{aligned}
C(S)=\max & \gamma_{S}(\lambda, \mu, \pi, \phi, \psi) \\
\text { s.t. } & \mu_{t}, \pi_{t}, \phi_{t}, \psi_{t} \geq 0, t=1,2, \ldots, T, \lambda_{T+1}=0 .
\end{aligned}
$$

Let $\left(\lambda^{*}, \mu^{*}, \pi^{*}, \phi^{*}, \psi^{*}\right)$ be optimal for the dual problem (3) with $S=N$. Then, again the duality theorem implies that

$$
\begin{aligned}
C(N)=\min & L_{N}\left(z, \mathbf{x}, \mathbf{y}, \mathbf{I}, \mathbf{q}, \lambda^{*}, \mu^{*}, \pi^{*}, \phi^{*}, \psi^{*}\right) \\
\text { s.t. } & \left(\mathbf{I}^{j}, \mathbf{q}^{j}\right) \in X^{j}, j \in N .
\end{aligned}
$$

Define for $j \in N$,

$$
\begin{array}{cl}
l_{j}=\min & \sum_{t=1}^{T}\left[h_{t}^{j} I_{t}^{j}+u_{t}^{j} q_{t}^{j}+\lambda_{t}^{*} q_{t}^{j}\right] \\
\text { s.t. } & \left(\mathbf{I}^{j}, \mathbf{q}^{j}\right) \in X^{j} .
\end{array}
$$

We claim that $\left(l_{1}, l_{2}, \ldots, l_{n}\right)$ is in the core of the cooperative game $(N, C)$.

THEOREM 1: The vector $\mathbf{I}=\left(l_{1}, l_{2}, \ldots, l_{n}\right)$ is in the core of the cooperative game $(N, C)$.

PROOF: Notice that in the optimization problem (4), no constraint is imposed on the decision variables $z, \mathbf{x}, \mathbf{y}$ and $\mathbf{I}^{0}$. Therefore, at the optimal solution $\left(z^{*}, \mathbf{x}^{*}, \mathbf{y}^{*}, \mathbf{I}^{*}, q^{*}\right)$, we must have that

$$
\begin{array}{r}
v=\sum_{t=1}^{T} \mu_{t}^{*}, \quad \lambda_{t}^{*}+\phi_{t}^{*}=c_{t}+\mu_{t}^{*}, \quad \lambda_{t}^{*}+\psi_{t}^{*}=o_{t}, \\
h_{t}^{0}=\lambda_{t+1}^{*}-\lambda_{t}^{*}+\pi_{t}^{*} .
\end{array}
$$

Thus,

$$
\begin{aligned}
\sum_{j \in N} l_{j} & =\sum_{t=1}^{T} \sum_{j \in N}\left[h_{t}^{j} I_{t}^{j *}+u_{t}^{j} q_{t}^{j *}+\lambda_{t}^{*} q_{t}^{j *}\right] \\
& =\min L_{N}\left(z, \mathbf{x}, \mathbf{y}, \mathbf{I}, \mathbf{q}, \lambda^{*}, \mu^{*}, \pi^{*}, \phi^{*}, \psi^{*}\right)=C(N) .
\end{aligned}
$$

On the other hand, for any $S \subset N$,

$$
\begin{aligned}
\sum_{j \in S} l_{j} & =\sum_{t=1}^{T} \sum_{j \in S}\left[h_{t}^{j} I_{t}^{j *}+u_{t}^{j} q_{t}^{j *}+\lambda_{t}^{*} q_{t}^{j *}\right] \\
& =\min L_{S}\left(z, \mathbf{x}, \mathbf{y}, \mathbf{I}, \mathbf{q}, \lambda^{*}, \mu^{*}, \pi^{*}, \phi^{*}, \psi^{*}\right) \\
& =\gamma_{S}\left(\lambda^{*}, \mu^{*}, \pi^{*}, \phi^{*}, \psi^{*}\right) \leq C(S) .
\end{aligned}
$$

This completes the proof.

We comment that Theorem 1 still holds when the retail stores' specific holding costs and transportation costs are convex by using convex programming duality theory (see, for instance, Bertsekas [4, p. 299]).

We now provide some intuition on the dual-variables and their relationships specified in (5). The first equation in (5) implies that the capacity investment cost is allocated to each period and $\mu_{t}^{*}$ is the capacity cost period $t$ pays. The dualvariable $\lambda_{t}^{*}$ specifies the charge for each unit product sent to the retail stores at period $t$. The products sent to the retail stores at period $t$ may either be produced at period $t$, outsourced at period $t$, or carried over from period $t-1$ to period $t$. If the products are produced at period $t$, the unit 
charge $\lambda_{t}^{*}$ equals the unit production cost plus the capacity cost for period $t$ (note that from the complementarity slackness condition $\phi_{t}^{*}=0$ as $x_{t}^{*}>0$ ). If the products come from outsourcing at period $t$, then the unit charge $\lambda_{t}^{*}$ equals the unit outsourcing cost (note that from the complementarity slackness condition $\psi_{t}^{*}=0$ since $y_{t}^{*}>0$ ). Finally, if the products are carried over from period $t-1$ to period $t$, then the unit charge $\lambda_{t}^{*}$ equals the unit charge $\lambda_{t-1}^{*}$ plus the unit inventory holding cost $h_{t-1}^{0}$ (note that from the complementarity slackness condition $\pi_{t-1}^{*}=0$ as $I_{t-1}^{*}>0$ ).

The definition of $l_{j}$ implies that each retail store shares a portion of the cost incurred at the production facility in addition to its own holding and transportation cost. The cost at the production facility is shared based on some dual solutions. Thus, the core allocation $\mathbf{I}$ belongs to the so-called Owen set defined by Owen [14], a set of allocations derived from optimal dual-solutions of certain dual problems. We show that the Owen set of $(N, C)$ may not be a singleton in general. To see this, consider a two-period model consisting of three players with no outsourcing, that is, $o_{t}$ is very large. We assume that both the production facility and the retail stores do not hold inventory and the production cost and the transportation cost are zero. The capacity installation cost is $c(z)=z$. Let the total demand at period one be $d_{1}$ and the total demand at period two be $d_{2}$. It is clear that the optimal capacity level is given by $z=\max \left(d_{1}, d_{2}\right)$. Assume that $S=\{1,2\}, S^{\prime}=\{1,3\}$, and $d^{1}=(0,2)$, $d^{2}=d^{3}=(1,0)$. For any $\left(\mu_{1}^{*}, \mu_{2}^{*}\right)$ with $\mu_{1}^{*}+\mu_{2}^{*}=1$ and $\mu_{1}^{*} \geq \mu_{2} \geq 0$, we can define $\phi^{*}=(0,0), \lambda^{*}=\mu^{*}$, $w_{1}^{*}=\lambda_{1}^{*}-\lambda_{2}^{*}$, and $\psi_{t}^{*}=o_{t}-\lambda_{t}^{*}$ such that (5) and the complementarity slackness conditions hold, which implies that $\left(\lambda^{*}, \mu^{*}, \pi^{*}, \phi^{*}, \psi^{*}\right)$ is optimal for problem (4). Thus, the set of allocations $\left\{\left(2 \mu_{2}^{*}, \mu_{1}^{*}, \mu_{1}^{*}\right): \mu_{1}^{*}+\mu_{2}^{*}=1, \mu_{1}^{*} \geq \mu_{2} \geq 0\right\}$ is a subset of the Owen set, which is clearly not a singleton.

\section{NONLINEAR COSTS AT THE PRODUCTION FACILITY}

In this section, we analyze capacity investment games in which costs at the production facility may be nonlinear. We restrict some of the cost functions to the following function class.

$$
\begin{aligned}
\mathcal{F}=\left\{f(\cdot): \mathfrak{R}^{+} \rightarrow \mathfrak{R} \mid f(0)=\right. & 0, f(x) / x \text { is non-increasing } \\
& f \text { is lower semi-continuous }\}
\end{aligned}
$$

In particular, we make the following assumption regarding the capacity investment cost function $v(z)$.

ASSUMPTION 1: The capacity investment $\operatorname{cost} v(\cdot) \in \mathcal{F}$.
The above assumption on the capacity investment cost $v(z)$ implies that the higher the capacity level, the lower the average unit capacity investment cost. Given this assumption, the retail stores may find it beneficial to form coalitions to build large capacity and make a joint production and distribution plan.

It is worth pointing out that we do not require function $v(z)$ to be either convex, concave, or monotone. In fact, $v(z)$ may not even be continuous. However, for technical reasons, we do assume that $v(z)$ is lower semicontinuous, that is, $\liminf _{y \rightarrow x} v(y) \geq v(x)$. With this assumption, we can show that the optimization models to be analyzed have optimal solutions. One important instance of $v(z)$ consists of a setup component and a variable cost component. Such a function is commonly used in practice to model economies of scale. Of course, our assumption covers more general function classes. For example, $v(z)$ may be a general concave function or an all-units discount cost function.

The generality of our assumption imposes a significant challenge in the analysis. Indeed, for a general function $v(z)$ satisfying Assumption 1, the optimization problem (1) may be rather complex, which makes the analysis of the resulting cooperative game $(N, C)$ rather difficult. To deal with this difficulty, we review and slightly extend in the next subsection a general technique developed in Chen [5] for a broad class of cooperative games with quantity discount.

\subsection{A Technique for Games with Quantity Discount}

In this subsection, we review and slightly extend the technique developed by Chen [5]. For this purpose, we digress for a second from the capacity investment game and consider a more general formulation of a cooperative game $(N, C)$ in which the characteristic function is defined by a two-stage optimization problem.

In the first stage, for a coalition $S$ in the cooperative game $(N, C)$, we make a single decision $y$ at a cost $c(y)$. The decision variable $y$ is allowed to take values in an interval $[0, a(S)]$, where $a(S)$ is a nonnegative scalar depending on $S$. After the first stage decision $y$ is made, the coalition $S$ will make another decision to achieve a second-stage minimal cost of the coalition, denoted as $g(y, S)$, which only depends on the first decision $y$ and the coalition $S$. The characteristic cost function $C$ of the cooperative game $(N, C)$ is then defined as follows.

$$
C(S)=\min _{0 \leq y \leq a(S)} c(y)+g(y, S) .
$$

Later on, we will see how the second-stage cost $g(y, S)$ is defined in the capacity investment game.

We are interested in cooperative games in which $c(y)$ exhibits economies of scale. Specifically, we assume that $c(\cdot) \in \mathcal{F}$. If $c(y)$ represents a production cost function, the 
assumption implies that the higher the production quantity, the lower the average unit production cost. Unfortunately, under this assumption the optimization problem (6) may be rather challenging and the duality approach for convex minimization problems employed in the previous section may not apply.

To overcome this challenge, we construct a new game $\left(N, C_{\hat{c}}\right)$, which is derived by replacing the cost function $c(y)$ in $(N, C)$ by a linear cost $\hat{c} y$. That is, the characteristic function of the cooperative game $\left(N, C_{\hat{c}}\right)$ is defined as follows.

$$
C_{\hat{c}}(S)=\min _{0 \leq y \leq a(S)} \hat{c} y+g(y, S) .
$$

Rather than analyzing the original game $(N, C)$, we focus on the game $\left(N, C_{\hat{c}}\right)$. We will prove that there exists a scalar $\hat{c}$ such that the new game $\left(N, C_{\hat{c}}\right)$ is dominated by $(N, C)$ in the sense that $C_{\hat{c}}(N)=C(N)$ while $C_{\hat{c}}(S) \leq C(S)$ for any $S \subset N$.

We make the following technical assumptions.

ASSUMPTION 2: a. $g(y, S)$ is lower semicontinuous in $y$ for any $S \subset N$.

b. $\lim _{y \rightarrow \infty} c(y)+g(y, S)=\infty$ if $a(S)=\infty$.

c. $y=0$ is an optimal solution for the optimization problem $\min _{0 \leq y \leq a(S)} \hat{c} y+g(y, S)$ for $\hat{c}$ sufficiently large.

Assumptions (a) and (b) guarantee the existence of an optimal solution for problem (6). Assumption (c) allows us to exclude some pathological cases.

Our analysis requires the existence of nondecreasing optimal solutions for problems (6) and (7). That is, there exists an optimal solutions $y^{*}(S)$ and $y_{\hat{c}}^{*}(S)$ for problems (6) and (7) parameterized by $S$, respectively, such that $y^{*}(S) \leq y^{*}(T)$ and $y_{\hat{c}}^{*}(S) \leq y_{\hat{c}}^{*}(T)$ for any $S \subset T$.

The following result is key to the analysis of this article.

PROPOSITION 1: Assume that $c(\cdot) \in \mathcal{F}$. If problems (6) and (7) have nondecreasing optimal solutions, then there exists a scalar $\hat{c}$ such that the cooperative game $\left(N, C_{\hat{c}}\right)$ is dominated by $(N, C)$. In this case, any core allocation for $\left(N, C_{\hat{c}}\right)$ is a core allocation for $(N, C)$.

A similar result is presented in Chen [5] for inventory centralization games with quantity discount, in which $a(S)=$ $+\infty$. The proof can be extended to the general setting here with minor modification and thus is omitted.

The proof of Proposition 1 implies that a core allocation of $(N, C)$ can be constructed in three steps (as long as the assumptions in Proposition 1 hold). First, compute

$$
C(N)=\min _{0 \leq y \leq a(N)} c(y)+g(y, N) .
$$

Second, find a constant $\hat{c}$ such that

$$
C(N)=C_{\hat{c}}(N)=\min _{0 \leq y \leq a(N)} \hat{c} y+g(y, N) .
$$

Third, find a core allocation for the game $\left(N, C_{\hat{c}}\right)$, which automatically gives a core allocation for $(N, C)$ according to Proposition 1.

The next two subsections will establish conditions under which Proposition 1 is applicable for the case with no inventory carryover and the case with inventory carryover, respectively.

\subsection{No Inventory Carry Over}

In this subsection, we analyze the capacity investment game for which no inventory is carried over from one period to the next at both the production facility and the retail stores. As we mentioned in the introduction, the assumption is appropriate for service industries in which holding inventory is impossible. With this assumption, we can drop the inventory terms in the optimization problem (1). In addition, the amount transported to each retail store is exactly the demand of the retail store. In this case, the transportation cost terms are constant and can also be dropped. Thus, the optimization problem (1) reduces to the following formulation.

$$
\begin{array}{cl}
\hat{f}(\mathbf{d})=\min & v(z)+\sum_{t=1}^{T}\left(c_{t}\left(x_{t}\right)+o_{t}\left(d_{t}-x_{t}\right)\right) \\
\text { s.t. } & x_{t}-z \leq 0, \quad t=1,2, \ldots, T, \\
& x_{t} \leq d_{t}, \quad t=1,2, \ldots, T, \\
& x_{t} \geq 0, \quad t=1,2, \ldots, T .
\end{array}
$$

The characteristic cost function is now defined as: $C(S)=$ $f\left(\sum_{j \in S} \mathbf{d}^{j}\right)$. Note that the game $(N, C)$ is not concave even when outsourcing is not allowed as illustrated by the example at the end of Section 2.

In the following, we prove that when the outsourcing cost functions are linear, there exists an optimal solution for problem (8) which is nondecreasing in $\mathbf{d}$.

LEMMA 1: Assume that the outsourcing cost $o_{t}(\cdot)$ is linear for $t=1,2, \ldots, T$. Then, there exists an optimal solution $\left(z^{*}(\mathbf{d}), x^{*}(\mathbf{d})\right)$ for problem (8) such that $\left(z^{*}(\mathbf{d}), x^{*}(\mathbf{d})\right)$ is nondecreasing in $\mathbf{d}$, i.e. for $\mathbf{d} \leq \mathbf{d}^{\prime},\left(z^{*}(\mathbf{d}), x^{*}(\mathbf{d})\right) \leq$ $\left(z^{*}\left(\mathbf{d}^{\prime}\right), x^{*}\left(\mathbf{d}^{\prime}\right)\right)$ componentwise.

PROOF: The objective function in (8) is submodular in $(z, \mathbf{x}, \mathbf{d})$, as it is a separable function and thus submodular (see Topkis [18] Theorem 2.6.4 or Simchi-Levi et al. [16] Theorem 2.3.3). Furthermore, the feasible set is a sublattice in $\Re \times \Re^{T} \times \Re^{T}$ (see Topkis [18] page 26). Thus, problem 
(8) is a minimization of a submodular function over a sublattice. This implies that the optimization problem (8) admits an optimal solution $\left(z^{*}(\mathbf{d}), x^{*}(\mathbf{d})\right)$ which is nondecreasing in $\mathbf{d}$ (see Theorem 2.8.3 in Topkis [18] or Theorem 2.3.7 in Simchi-Levi et al. [16]).

It is worth mentioning that the above lemma is true for any function $v(\cdot)$ and $c_{t}(\cdot)$ as long as optimal solutions exist.

We are now ready to present our main result for the case with no inventory carryover.

THEOREM 2: Assume that $v(\cdot), c_{t}(\cdot) \in \mathcal{F}$ and $o_{t}(\cdot)$ is linear for $t=1,2, \ldots, T$. Then, the capacity investment game $(N, C)$ without inventory carryover is balanced.

PROOF: First, define

$$
\begin{array}{cl}
g(z, S)=\min & \sum_{t=1}^{T}\left(c_{t}\left(x_{t}\right)+o_{t}\left(d_{t}^{S}-x_{t}\right)\right) \\
\text { s.t. } & x_{t}-z \leq 0, t=1,2, \ldots, T, \\
& x_{t} \leq d_{t}^{S}, \quad t=1,2, \ldots, T, \\
& x_{t} \geq 0, t=1,2, \ldots, T,
\end{array}
$$

where $d_{t}^{S}=\sum_{j \in S} d_{t}^{j}$. We have that $C(S)=\min _{z \geq 0} v(z)+$ $g(z, S)$. Proposition 1 together with Lemma 1 implies that we can construct a new game with $v(\cdot)$ replaced by a linear function which is dominated by the game $(N, C)$. Thus, it suffices to show that the new game has a nonempty core.

Similarly, for a given period $t_{0}$ with $x_{t_{0}}$, define

$$
\begin{aligned}
g_{t_{0}}\left(x_{t_{0}}, S\right)=\min \quad & v(z)+o\left(d_{t_{0}}^{S}-x_{t_{0}}\right) \\
& +\sum_{t=1: T, t \neq t_{0}}\left(c_{t}\left(x_{t}\right)+o_{t}\left(d_{t}^{S}-x_{t}\right)\right) \\
\text { s.t. } \quad & x_{t}-z \leq 0, t=1,2, \ldots, T, \\
& x_{t} \leq d_{t}^{S}, \quad t=1,2, \ldots, T, t \neq t_{0} \\
& x_{t} \geq 0, \quad t=1,2, \ldots, T, t \neq t_{0} .
\end{aligned}
$$

Then, we have that $C(S)=\min _{0 \leq x_{t_{0}} \leq d_{t_{0}}^{S}} c_{t_{0}}\left(x_{t_{0}}\right)+g_{t_{0}}\left(x_{t_{0}}, S\right)$. Thus, Proposition 1 together with Lemma 1 implies that we can construct a new game with $c_{t_{0}}(\cdot)$ replaced by a linear function which is dominated by the game $(N, C)$.

The above discussion implies that we can construct a new game with $v(\cdot)$ and $c_{t}(\cdot)$ all replaced by linear functions such that the new game is dominated by $(N, C)$. Hence, the core of $(N, C)$ includes the core of the new game as a subset, which is nonempty according to Theorem 1 .

As we pointed out earlier, when $v(\cdot), c_{t}(\cdot)$ and/or $o_{t}(\cdot)$ are convex, the characteristic cost function $C(S)$ may not even be subadditive and thus the game $(N, C)$ may have an empty core. One may wonder whether Theorem 2 still holds if the outsourcing costs $o_{t}(\cdot)$ also belong to the function class $\mathcal{F}$ instead of being linear. Unfortunately, the answer is negative even when the outsourcing costs $o_{t}(\cdot)$ involve only setup cost components and $v(\cdot)$ and $c_{t}(\cdot)$ are linear. We illustrate this in the following example. Let

$$
T=2, \quad v(z)=z, \quad c_{t}\left(x_{t}\right)=0, \quad o_{t}\left(y_{t}\right)=\delta\left(y_{t}\right),
$$

where $\delta(u)=1$ if $u>0$ and 0 otherwise. For a given $z$, it is clear that the optimal production quantities $x_{t}=\min \left(d_{t}, z\right)$ and the optimal outsourcing quantities $y_{t}=\max \left(d_{t}-z, 0\right)$. Thus, problem (8) is reduced to the following:

$$
\min _{z \geq 0} z+\delta\left(d_{1}-z\right)+\delta\left(d_{2}-z\right) .
$$

The optimal objective value and the optimal capacity level are given as follows (note that at the boundaries, we have multiple optimal solutions).

$$
\begin{aligned}
& \left(\hat{f}\left(d_{1}, d_{2}\right), z^{*}\left(d_{1}, d_{2}\right)\right) \\
& =\left\{\begin{array}{lc}
\left(\max \left(d_{1}, d_{2}\right), \max \left(d_{1}, d_{2}\right)\right), & \left|d_{1}-d_{2}\right| \leq 1,0 \leq d_{1} \\
\left(1+d_{1}, d_{1}\right), & \leq 2,0 \leq d_{2} \leq 2, \\
\left(1+d_{2}, d_{2}\right), & d_{2}-d_{1} \geq 1,0 \leq d_{1} \leq 1, \\
(2,0), & d_{1}-d_{2} \geq 1,0 \leq d_{2} \leq 1,
\end{array}\right.
\end{aligned}
$$

We now consider a game with three players with $\mathbf{d}^{1}=$ $(0,1.5-\epsilon), \mathbf{d}^{2}=(1-\epsilon, 0.5-\epsilon)$ and $\mathbf{d}^{3}=(\epsilon, 2 \epsilon)$, where $\epsilon$ is a small positive number. The characteristic cost function is specified as follows.

$$
\begin{aligned}
C(\{1\})=1, C(\{2\})=1-\epsilon, C(\{3\}) & =2 \epsilon, \\
C(\{1,2\})=2-2 \epsilon, C(\{1,3\})=1+\epsilon, C(\{2,3\}) & =1, \\
C(\{1,2,3\}) & =2 .
\end{aligned}
$$

If $\left(l_{1}, l_{2}, l_{3}\right)$ is in the core of $(\{1,2,3\}, C)$, then $l_{1}+l_{2}+l_{3}=2$, which implies that $l_{3}=2-l_{1}-l_{2}$. Therefore, the inequalities $l_{1} \leq C(\{1\})=1$ and $l_{2}+l_{3}=2-l_{1} \leq C(\{2,3\}) \leq 1$ imply that $l_{1}=1$. Similarly, the inequalities $l_{2} \leq C(\{2\})=1-\epsilon$ and $l_{1}+l_{3}=2-l_{2} \leq C(\{1,3\})=1+\epsilon$ imply that $l_{2}=1-\epsilon$. However, $l_{1}+l_{2}=2-\epsilon>2-2 \epsilon=C(\{1,2\})$, violating the requirement that $l_{1}+l_{2} \leq C(\{1,2\})$ in the core definition. Thus, the core of $(\{1,2,3\}, C)$ is empty. Of course, this implies that in this case Lemma 1 fails for the concave outsourcing costs $o_{t}(\cdot)$. As an illustration, observe that $z^{*}\left(d_{1}, d_{2}\right)$ is not monotone in $d_{1}$ for a given $d_{2} \in(1,2)$.

\subsection{Inventory Carry Over}

In this subsection, we assume that the production facility and all the retail stores can carry inventory and the capacity 
investment cost $v(\cdot) \in \mathcal{F}$. Similar to the previous subsection, we apply Proposition 1 to construct a new game with a linear investment cost that is dominated by $(N, C)$. For this purpose, we illustrate in the following lemma that under certain assumptions, the higher the demand, the larger the optimal capacity level. This monotonicity result plays an important role in our analysis. Interestingly, this result is true for any capacity investment cost function as long as the optimal solution for problem (1) exists.

LEMMA 2: Assume that all costs except the capacity investment cost are linear for $t=1,2, \ldots, T$ and $j \in N$. There exists an optimal capacity level $z(\mathbf{D})$ for problem (1) with demand profile $\mathbf{D}=\left(\mathbf{d}^{1}, \mathbf{d}^{2}, \ldots, \mathbf{d}^{n}\right)$ such that $z(\mathbf{D})$ is nondecreasing in $\mathbf{D}$ componentwise.

PROOF: Recall the function $g(z, \mathbf{D})$ defined in (8), which represents the minimum cost of production, outsourcing, inventory holding, and transportation of satisfying the demand profile $\mathbf{D}$ for a given capacity level $z$. Let $z(\mathbf{D})$ be the maximum optimal solution for $\min _{z \geq 0} v(z)+g(z, \mathbf{D})$. To prove that $z(\mathbf{D})$ is nondecreasing in $\mathbf{D}$, it suffices to show that $g(z, \mathbf{D})$ is submodular in $\left(z, d_{t_{0}}^{j_{0}}\right)$ for any $t_{0}=1,2, \ldots, T$ and $j_{0} \in N$ (from Theorem 2.8.3 in Topkis [18] or Theorem 2.3.7 in Simchi-Levi et al. [16]). For this purpose, we construct a minimum-cost network flow problem in which the cost functions on some arcs depend on parameters representing the production capacity or demand and then employ Theorem 17 in Granot and Veinott [10]. To use the theorem, we temporarily assume that the production capacities at different periods can be different and are denoted as $z_{t}$ for period $t$.

We now construct a network $G=(V, E)$. In this network, the set of vertices $V$ is given as follows: vertices $(j, t)$ representing retail store $j$ (the production facility if $j=0$ ) at time $t, j \in N \cup\{0\}$, and $t=1, \ldots, T$; two vertices $(0,+)$ and $(0,-)$ representing production and outsourcing, respectively; and an artificial node $(0,0)$.

The arc set $E$ is given as follows. There are directed arcs from $(0,+)$ and $(0,-)$ to $(0, t)$, whose flows correspond to the production quantity $x_{t}$ and the outsourcing quantity $y_{t}$, respectively. There is a directed arc from $(j, t)$ to $(j, t+1)$, whose flow corresponds to the inventory $I_{t}^{j}$ carried over from period $t$ to period $t+1$ for retailer $j$ (the production facility if $j=0)$. There is a directed arc from $(0, t)$ to $(j, t)$, whose flow corresponds to the inventory $q_{t}^{j}$ transhipped from the production facility to retailer $j$ at time period $t$. There is a directed arc from node $\left(j_{0}, t_{0}\right)$ to the artificial node $(0,0)$ representing demand $d_{t_{0}}^{j_{0}}$. Finally, there are directed arcs from $(0,0)$ to $(0,+)$ and $(0,-)$, respectively.

At each vertex, there is a given demand. Specifically, at vertex $(j, t)$ with $(j, t) \neq\left(j_{0}, t_{0}\right)$ and $j \neq 0$, the demand is $d_{t}^{j}$, and the demand at $(0,0)$ is $-\sum_{t=1: T, j \in N} d_{t}^{j}+d_{t_{0}}^{j_{0}}$. The demand is zero at other vertices including $\left(j_{0}, t_{0}\right)$.
For each arc, there is a cost which may depend on its flow and some parameters. Specifically, the cost on the arc from $(0,+)$ to $(0, t)$ is specified as $c_{t}\left(x_{t}\right)+\delta\left(0 \leq x_{t} \leq z_{t}\right)$ given its flow $x_{t}$, where $\delta(\cdot)$ is an indicator taking value 0 if the statement inside $\delta$ is true and value $\infty$ otherwise (notice that the $\operatorname{cost}$ term $\delta\left(0 \leq x_{t} \leq z_{t}\right)$ implies that the flow on the arc from $(0,+)$ to $(0, t)$ is nonnegative and cannot exceed the production capacity $\left.z_{t}\right)$; the cost on the arc from $(0,-)$ to $(0, t)$ is $O_{t}\left(y_{t}\right)+\delta\left(y_{t} \geq 0\right)$ given its flow $y_{t}$; the cost on the arc from $(j, t)$ to $(j, t+1)$ is $h_{t}^{j}\left(I_{t}^{j}\right)+\delta\left(I_{t}^{j} \geq 0\right)$ given its flow $I_{t}^{j}$; the cost on the arc from $(0, t)$ to $(j, t)$ is $u_{t}^{j}\left(q_{t}^{j}\right)+\delta\left(q_{t}^{j} \geq 0\right)$; and the cost on the arc from $\left(j_{0}, t_{0}\right)$ to $(0,0)$ is $\delta\left(f=d_{t_{0}}^{j_{0}}\right)$ given its flow $f$, or equivalently, the flow must be $d_{t_{0}}^{j_{0}}$; and the costs on the arcs from $(0,0)$ to $(0,+)$ and $(0,-)$ are zero.

The minimum cost of the above network flow problem depends on $z_{t}$ for $t=1,2, \ldots, T$ and the demand profile $\mathbf{D}$ and is denoted as $\hat{g}\left(z_{1}, \ldots, z_{T}, \mathbf{D}\right)$. Notice that $g(z, \mathbf{D})=$ $\hat{g}(z, \ldots, z, \mathbf{D})$.

We now consider two arcs in the network flow problem: the arc from $(0,+)$ to $(0, t)$ and the $\operatorname{arc}$ from $\left(j_{0}, t_{0}\right)$ to $(0,0)$. Clearly, these two arcs are complements. That is, any simple cycle containing the two arcs orients them in the same direction. Fix the parameters $\left(z_{1}, \ldots, z_{T}\right)$ and $\mathbf{D}$ except $z_{t}$ and $d_{t_{0}}^{j_{0}}$. Notice that our assumption implies that all the cost functions are convex in arc flows. In addition, the cost functions on the arc from $(0,+)$ to $(0, t)$ and the arc from $\left(j_{0}, t_{0}\right)$ to $(0,0)$ are submodular in $\left(x_{t}, z_{t}\right)$ and $\left(f, d_{t_{0}}^{j_{0}}\right)$, respectively. From Theorem 17 of Granot and Veinott [10], the minimum cost $\hat{g}\left(z_{1}, \ldots, z_{T}, \mathbf{D}\right)$ is submodular in $\left(z_{t}, d_{t_{0}}^{j_{0}}\right)$ for any $t, t_{0}=1,2 \ldots, T$.

We now prove that $g(z, \mathbf{D})=\hat{g}(z, \ldots, z, \mathbf{D})$ is submodular in $\left(z, d_{t_{0}}^{j_{0}}\right)$ for any $t_{0}=1,2 \ldots, T$ and $j \in N$. To show this, consider $z<\hat{z}$ and $d_{t_{0}}^{j_{0}}>\hat{d}_{t_{0}}^{j_{0}}$. Let $\hat{\mathbf{D}}$ be a demand profile derived from $\mathbf{D}$ by replacing $d_{t_{0}}^{j_{0}}$ in $\mathbf{D}$ with $\hat{d}_{t_{0}}^{j_{0}}$. We have that

$$
\begin{aligned}
g(z, \mathbf{D})-g(z, \hat{\mathbf{D}}) & =\hat{g}(z, \ldots, z, \mathbf{D})-\hat{g}(z, \ldots, z, \hat{\mathbf{D}}) \\
& \geq \hat{g}(z, \ldots, z, \hat{z}, \mathbf{D})-\hat{g}(z, \ldots, z, \hat{z}, \hat{\mathbf{D}}) \\
& \geq \hat{g}(z, \ldots, z, \hat{z}, \hat{z}, \mathbf{D})-\hat{g}(z, \ldots, z, \hat{z}, \hat{z}, \hat{\mathbf{D}}) \\
& \geq \ldots \\
& \geq \hat{g}(\hat{z}, \ldots, \hat{z}, \mathbf{D})-\hat{g}(\hat{z}, \ldots, \hat{z}, \hat{\mathbf{D}}) \\
& =g(\hat{z}, \mathbf{D})-g(\hat{z}, \hat{\mathbf{D}}),
\end{aligned}
$$

where the inequalities follow from the fact that $\hat{g}\left(z_{1}, \ldots\right.$, $\left.z_{T}, \mathbf{D}\right)$ is submodular in $\left(z_{t}, d_{t_{0}}^{j_{0}}\right)$ for any $t, t_{0}=1,2 \ldots, T$. The above inequalities imply that $g(z, \mathbf{D})$ is submodular in $\left(z, d_{t_{0}}^{j_{0}}\right)$ and thus the optimal capacity level $z(\mathbf{D})$ is nondecreasing in $\mathbf{D}$ componentwise (again from Theorem 2.8.3 in Topkis [18] or Theorem 2.3.7 in Simchi-Levi et al. [16]). 
It is appropriate to point out that Lemma 2 still holds when the inventory holding costs $h_{t}^{j}(\cdot)$ and the transportation costs $u_{t}^{j}(\cdot)$ are convex for $t=1,2, \ldots, T$ and $j \in N$.

We are now ready to present our main result of this subsection.

THEOREM 3: Assume that $v(\cdot) \in \mathcal{F}$ and all other costs are linear. Then, the capacity investment game $(N, C)$ with inventory carryover is a balanced game. Moreover, a core allocation can be computed by applying the procedure outlined in Subsection 4.1 .

Lemma 2 and Proposition 1 imply that the capacity investment game $(N, C)$ is dominated by a new game with linear capacity investment cost, which from Theorem 1 has an allocation in the core that can be computed by employing linear programming duality theory. Therefore, $(N, C)$ has an allocation in the core as well, which can be computed by applying the procedure outlined in Subsection 4.1.

Theorem 3 is proven under the assumption that all costs except the capacity investment cost are linear. It is still valid when the inventory holding costs $h_{t}^{j}(\cdot)$ and the transportation costs $u_{t}^{j}(\cdot)$ are convex for $t=1,2, \ldots, T$ and $j \in N$. As we pointed out earlier, it is common to assume linear inventory holding costs and outsourcing costs in the operations literature, while the assumption of linear production cost is appropriate for settings in which economies of scale are negligible or enormous so that the setup only happens at the beginning of the planning horizon. When $c_{t}(\cdot), h_{t}^{0}(\cdot)$ and/or $o_{t}(\cdot)$ are convex, the core of $(N, C)$ may be empty as we pointed out earlier. When the outsourcing cost functions $o_{t}(\cdot)$ are concave, the core of $(N, C)$ may be empty as well as illustrated by the example in Subsection 4.2 (we can choose extremely high inventory holding costs $h_{t}^{j}(\cdot)$ so that the model analyzed here reduces to the one without inventory carryover in Subsection 4.2).

It remains open whether the core of $(N, C)$ is nonempty if $o_{t}(\cdot)$ are linear and $c_{t}(\cdot)$ and $h_{t}^{0}(\cdot)$ are concave. In this case, the technique used to prove Theorem 3 may not apply anymore. In fact, we show by an example that the monotonicity of the optimal capacity level does not hold even when the production costs involve only fixed cost components, outsourcing is not allowed, and the retail stores do not hold any inventory. Let

$$
\begin{gathered}
T=3, \quad v(z)=\kappa z, \quad h_{t}^{0}=0 \\
c_{t}\left(x_{t}\right)=\delta\left(x_{t}\right), \quad t=1,2,3
\end{gathered}
$$

and

$$
d_{1}=d_{2}=1, \quad d_{3}=1+\eta
$$

If the production is made at one period, then the capacity is $3+\eta$ and the total cost is $\kappa(3+\eta)+1$; if the production is made at two periods, then the capacity is $(3+\eta) / 2$ and the total cost is $\kappa(3+\eta) / 2+2$; if the production is made at three periods, then the capacity is $(3+\eta) / 3$ and the total cost is $\kappa(3+\eta) / 3+3$. For $\kappa=1 / 2$, it is optimal to set the capacity level at $3+\eta$ when $\eta \in[0,1]$, the capacity level at $(3+\eta) / 2$ when $\eta \in[1,9]$ and the capacity level at $(3+\eta) / 3$ when $\eta \geq 9$. Thus, the optimal capacity level may decrease with demand.

We now present an example to illustrate how the procedure outlined in Subsection 4.1 can be used to derived a core allocation of $(N, C)$. Consider a two-period model consisting of three players with no outsourcing. We assume that both the production facility and the retail stores do not hold inventory and the production cost and the transportation cost are zero. The capacity installation cost is $c(z)=\min (z, 0.5+0.5 z)$. Let the total demand at period one be $d_{1}$ and the total demand at period two be $d_{2}$. It is clear that the optimal capacity level is given by $z=\max \left(d_{1}, d_{2}\right)$. Assume that $S=\{1,2\}$, $S^{\prime}=\{1,3\}$ and $d^{1}=(0,1), d^{2}=d^{3}=(1,0)$. For this simple game, we can directly derive the characteristic function and determine the core $(\{1,2,3\}, C)$. Specifically, we have

$$
\begin{array}{r}
C(\{1\})=C(\{2\})=C(\{3\})=C(\{1,2\})=C(\{1,3\})=1, \\
C(\{2,3\})=C(\{1,2,3\})=1.5,
\end{array}
$$

and the core of $(\{1,2,3\}, C)$ is

$$
\begin{aligned}
\left\{\left(l_{1}, l_{2}, l_{3}\right): l_{1}+l_{2}+l_{3}\right. & =1.5, l_{1}+l_{2} \leq 1, l_{1}+l_{3} \leq 1, \\
& \left.l_{2}+l_{3} \leq 1.5, l_{1} \leq 1, l_{2} \leq 1, l_{3} \leq 1\right\}
\end{aligned}
$$

which contains infinite number of allocations. We now apply the procedure outlined in Subsection 4.1. The first step of the procedure gives $C(\{1,2,3\})=1.5$. In the second step, we note that for any linear capacity installation cost $\hat{c}$, $C_{\hat{c}}(\{1,2,3\})=\hat{c} / 2$. Thus, $C_{\hat{c}}(\{1,2,3\})=C(\{1,2,3\})$ gives $\hat{c}=0.75$. Finally, in the third step, we have that

$$
\begin{aligned}
& C_{\hat{c}}(\{1\})=C_{\hat{c}}(\{2\})=C_{\hat{c}}(\{3\})=C_{\hat{c}}(\{1,2\}) \\
&=C_{\hat{c}}(\{1,3\})=0.75, C_{\hat{c}}(\{2,3\})=C_{\hat{c}}(\{1,2,3\})=1.5,
\end{aligned}
$$

and the core of $\left(\{1,2,3\}, C_{\hat{c}}\right)$ is the set

$$
\begin{array}{r}
\left\{\left(l_{1}, l_{2}, l_{3}\right): l_{1}+l_{2}+l_{3}=1.5, l_{1}+l_{2} \leq 0.75, l_{1}+l_{3} \leq 0.75\right. \\
\left.l_{2}+l_{3} \leq 1.5, l_{1} \leq 0.75, l_{2} \leq 0.75, l_{3} \leq 0.75\right\}
\end{array}
$$

Interestingly, it reduces to be a singleton $\{(0,0.75,0.75)\}$. Observe that retail store 1 adds zero cost to any coalition and retail stores 2 and 3 are symmetric. Thus, this core allocation 
is reasonable as it assigns a cost 0 to retail store 1 and an identical cost 0.75 to retail stores 2 and 3 .

\section{CONCLUDING REMARKS}

In this article, we analyze cost allocation of capacity investment games. In such a game, capacity is built at the beginning of the planning horizon, which is followed by the production/outsourcing and distribution of the product to different retail stores. Under various conditions, we show that a capacity investment game admits a nonempty core. These conditions assume that retail store specific costs (the transportation costs to a retail store and the inventory holding costs at the retail store) are convex while the capacity investment cost exhibits the quantity discount property and the other costs at the production facility are linear. In the special case with no inventory carryover, we can also allow the production cost at each period to exhibit the quantity discount property.

To prove the nonemptiness of the core of a capacity investment game with a general capacity cost, we construct another game with linear capacity cost. In the construction, we keep the minimum cost of the grand coalition unchanged. Meanwhile, the minimum cost for each coalition does not decrease. This construction allows one to claim that any allocation in the core of the newly constructed game is in the core of the original game with general capacity cost. This construction builds on and extends a technique developed in Chen [5] for inventory centralization games with general quantity discount. To apply the technique, we prove that the optimal capacity investment level is nondecreasing in terms of the demand vectors of the retail stores.

It would be interesting to extend our results and analysis to more general settings. Specifically, allowing cost functions (other than the capacity investment cost) to be concave remains a significant challenge. We have a complete understanding of the capacity investment game when inventory is not allowed to be carried over from one period to the next. However, when inventory can be carried over, all our results require linearity on production facility specific costs (other than the capacity investment cost) while the retail store specific costs are convex. We would like to see whether these conditions can be relaxed.

We are also interested in extending our model to settings with stochastic demand. If all costs are linear, we can employ the stochastic programming duality approach similar to the linear programming duality approach used in Section 3 to show the nonemptiness of the core. The results in Subsection 4.2 can also be extended. However, in general, the problem remains open.

Finally, in this article, we focus on the existence of core allocations and mechanisms of computing such allocations. It would be interesting to compare the derived core allocations with allocations based on other cooperative game concepts such as the Shapley value and the nucleolus.

\section{ACKNOWLEDGMENTS}

The authors thank Jiawei Zhang and Yuhan Zhang for their helpful comments. The current proof of Lemma 2 was suggested by Jiawei Zhang, which replaces the original proof based on repeated application of the max-flow min-cut theorem. The authors also thank Professor Federgruen, the AE, and three anonymous referees for valuable comments and suggestions.

This research was partially supported by the National Science Foundation Grants CMMI-0653909, CMMI-0926845 ARRA, CMMI-1030932, and the National Science Foundation of China grant 71228203 .

\section{REFERENCES}

[1] S. Anily and M. Haviv, The cost allocation problem for the first order interaction joint replenishment model, Oper Res 55(2) (2007), 292-302.

[2] S. Anily and M. Haviv, Cooperation in service systems, Oper Res 58(3) (2009), 660-673.

[3] A. Atamtürk and D.S. Hochbaum, Capacity acquisition, subcontracting, and lot sizing, Manag Sci 47(8) (2001), 1081-1100.

[4] D. Bertsekas, Nonlinear programming, Athena Scientific, Boston, 1995

[5] X. Chen, Inventory centralization games with price-dependent demand and quantity discount, Oper Res 57(6) (2009), 1394-1406.

[6] X. Chen and J. Zhang, A stochastic programming duality approach to inventory centralization games, Oper Res 57(4) (2009), 840-851.

[7] X. Chen and J. Zhang, Duality approaches to economic lot-sizing games, Working paper, New York University, 2006.

[8] I. Curiel, Cooperative game theory and applications: Cooperative games arising from combinatorial optimization problem, Kluwer Academic Publishers, Netherlands, 1997.

[9] M. Gopaladesikan, N. Uhan, and J. Zou, A primal-dual algorithm for computing a cost allocation in the core of economic lot-sizing games. working paper, Purdue University, 2011.

[10] F. Granot and A. Veinott, Substitutes, complements and ripples in network flows, Math Oper Res 10(3) (1985), 471-497.

[11] L.A. Guardiola, A. Meca, and J. Puerto, Production-inventory games: A new class of totally balanced combinatorial optimization games, Games Econ Behav 65(1) (2009), 205-219.

[12] J. Feigenbaum, C. Papadimitriou, and S. Shenker, "Sharing the cost of muliticast transmissions (preliminary version)," In STOC '00: Proceedings of the thirty-second annual ACM symposium on Theory of computing, Portalnd, OR, USA, 2000, pp. 218-227.

[13] M. Nagarajan and G. Sošić, Game-theoretical analysis of cooperation among supply chain agents: Review and extensions, Eur J Oper Res 187(3) (2008), 719-745.

[14] G. Owen, On the core of linear production games, Math Program 9 (1975), 358-370. 
[15] M. Shubik, Incentives, decentralized control, the assignment of joint costs and internal pricing, Manag Sci 8(3) (1962), 325-343.

[16] D. Simchi-Levi and X. Chen, The logic of logistics: Theory, algorithms, and applications for logistics and supply chain management, 2nd ed., Springer-Verlag, New York, 2005.

[17] A. Tamir, On the core of cost allocation games defined on location problems, Trans Sci 27(1) (1993), 81-86.

[18] D.M. Topkis, Supermodularity and complementarity, Princeton University Press, Princeton, NJ, 1998.

[19] W. van den Heuvel, P.E.M. Borm, and H. Hamers, Economic lot-sizing games, Euro J Oper Res 176(2) (2007), 1117-1130.

[20] Xu D. and R. Yang, A cost-sharing method for an economic lot-sizing game, Oper Res Lett 37(2) (2009), 107-110.
[21] H.P. Young, "Cost allocation," Handbook of game theory with economic applications, Volume 2, R.J. Aumann and S. Hart (Editors), 1st ed., Elsevier, 1994.

[22] H.P. Young, N. Okada, and T. Hashimoto, Cost allocation in water resources development, Water Resour Res 18(3) (1982), 463-475.

[23] Y. Yu, S. Benjaafar, and Y. Gerchak, On service capacity pooling and cost sharing among independent firms. Working paper, University of Minnesota, 2008.

[24] J. Zhang, Cost allocation for joint replenishment models, Oper Res 57(1) (2009), 146-156.

[25] J.M. Zolezzi and H. Rudnick, Transmission cost allocation by cooperative games and coalition formation, IEEE Trans Power Sys 17(4) (2002), 1008-1015. 